\title{
Macroscopic Findings Biospecimen Usability
}

National Cancer Institute

\section{Source}

National Cancer Institute. Macroscopic Findings Biospecimen Usability. NCI Thesaurus.

Code C119870.

A qualitative or quantitative measure that describes a biospecimen's suitability for macroscopic testing purposes. 